Contemporary Physics

Vol. 51, No. 5, Dec 2010, 1-18

\title{
The Astrophysical Environment of the Solar Birthplace
}

\author{
Jonathan P. Williams* \\ Institute for Astronomy, University of Hawaii, Honolulu , USA \\ (Received 00 Month 200x; final version received 00 Month 200x)
}

\begin{abstract}
Our Sun, like all stars, formed within a cold molecular cloud. Astronomical observations and theory provide considerable detail into this process. Yet cosmochemical observations of short lived radionuclides in primitive meteorites, in particular ${ }^{60} \mathrm{Fe}$, provide unequivocal evidence that the early solar system inherited fresh nucleosynthetic material from the core of a hot, massive star, almost certainly ejected in a supernova explosion. I give a short introduction to the fields of star formation and meteoritics and discuss how the reconciliation of their disparate clues to our origin places strong constraints on the environment of the Solar birthplace. Direct injection of supernova ejecta into a protoplanetary disk or a dense molecular core is unlikely since their small sizes require placement unusually close to the massive star. Lower density molecular cloud clumps can capture more ejecta but the radionuclides decay during the slow gravitational collapse. The most likely scenario is on the largest scales via the formation of enriched molecular clouds at the intersection of colliding supernova bubbles in spiral arms.
\end{abstract}

Keywords: interstellar medium — star formation — short lived radionuclides

\section{Introduction}

We know a great deal about the Sun. We know, to astonishing precision, its mass, size, composition, and age. Each of these measurements was a triumph of astrophysics. Nobel prize winning work has detailed the nuclear reactions in its core and its eventual fate as a white dwarf. Yet the origins of our Sun and of the solar system, are far less well understood. This is an active area of current research and, as with most areas of astronomy, one that is driven by technology and has exciting prospects ahead. Moreover, with the ever-growing catalog of extrasolar planets, and now direct imaging of multiple planet systems, the question of the characteristics of the solar system relative to others can begin to be addressed.

The Sun is only about one third the age of the Universe. We can observe distant galaxies that formed before our Sun existed. We can also observe nearby protostars as they condense from a diffuse cloud. By studying today's protostars and the disks around them, we can learn about the processes that led to the formation of our Sun and solar system. The underlying theoretical principles are described in [1, 2]. I outline the scales of mass, size, and time for the different stages of star formation in $\oint_{2}$,

We can also learn about the conditions of the early solar system by studying meteorites, the rocky remnants of the protosolar disk. The age of the solar system was deduced from radioactive isotopic analysis of the most primitive meteorites [3]. Cosmochemical studies of meteoritic mineralogy and composition reveal the way in which interstellar dust grains metamorphised to larger and larger pieces and, ultimately, planets. The decay products of short-lived radionuclides, those with half lives comparable to planet forming timescales, time stamp the evolution of the early solar system. Several short lived radionuclides appear to be abnormally abundant and provide critical clues to the environment of the solar birthplace. I describe these in 93 ,

By providing fine detail on small scale processes within one solar system (ours), cosmochemical studies are complementary to astronomical observations that show the gross properties of many

*Corresponding author. Email: jpw@ifa.hawaii.edu 
protostars and exoplanetary systems. Perhaps not surprisingly, given the enormous difference in scales and the intrinsic diversity of star and planet formation, the two fields do not always agree. Nevertheless, reconciling the different lines of evidence, as best as possible, has value in showing the limits of our current understanding and in placing our solar system into context with the hundreds of others now known to exist. In 4 , I show how the inferred abundance of the short lived radionuclide, ${ }^{60} \mathrm{Fe}$, in small primitive rocks forces us to consider the processes of star formation in the setting of the largest scales in the Galaxy, that of spiral arms. This paper is intended for a general audience. At about the same time, a more specialized astrophysical review of some of the topics raised here was being independently written and is currently in press [4].

\section{Star and planet formation}

\section{$2.1 \quad$ The interstellar medium}

Stars form from the gravitational collapse of the interstellar medium. This consists of gas, about $90 \%$ hydrogen and 10\% helium by number, with trace quantities of heavier elements which are mostly locked up in submicron sized dust grains. The dust accounts for about $1 \%$ of the mass of the interstellar medium and is produced mainly in the winds from evolved stars and, to a lesser extent, supernovae.

The astonishing precision to which I alluded to previously concerning the Sun does not apply to the interstellar medium. Whereas we know each of the Sun's mass, size and age to at least 4 significant figures, the properties of the interstellar medium are far less precisely measured with uncertainties of a factor of 2 or even more. This is due to the nature of the interstellar medium as a diverse collection of diffuse, inhomogeneous clouds with irregular (possibly fractal) boundaries and uncertain distances that are subject to varied physical influences and are generally far from thermodynamic equilibrium [5]. Nevertheless, the range of scales involved in star and planet formation are so vast that these rough measures are sufficient to elucidate the dominant physical mechanisms at work.

The interstellar medium exists in a wide range of temperatures, $T$, and number densities, $n$, in distinct phases in approximate pressure balance, $P=n k T \approx 10^{-13} \mathrm{~Pa}[\underline{6}]$. Most of the volume consists of hot, rarefied, ionised gas with $T \approx 10^{6} \mathrm{~K}, n \approx 5 \times 10^{3} \mathrm{~m}^{-3}$, heated by supernovae shock waves. Most of the mass is in atomic clouds with a range of temperatures $T \approx 10^{2}-10^{4} \mathrm{~K}$ and densities $n \approx 50-0.5 \times 10^{6} \mathrm{~m}^{-3}$ respectively. The coldest, densest regions of the interstellar medium are molecular with $T \approx 20 \mathrm{~K}, n \gtrsim 5 \times 10^{8} \mathrm{~m}^{-3}$, Because of their cool temperatures, the peak of the emission occurs at a wavelength $\lambda=h c / k T \simeq 1 \mathrm{~mm}$, and high frequency radio observations are required to observe the emission from this phase.

Molecular clouds come in a range of sizes and are the sites of all star formation. Small clouds dominate by number but large clouds by integrated mass. Using astronomical units for a solar mass, $1 M_{\odot}=2 \times 10^{30} \mathrm{~kg}$, and parsec, $1 \mathrm{pc}=3.09 \times 10^{16} \mathrm{~m}$, giant molecular clouds are those with masses $M \approx 10^{4}-10^{6} M_{\odot}$ and sizes $L \approx 10-100$ pc. These are the most massive objects in the Galactic disk and are where most stars are born.

Each phase of the interstellar medium is inhomogeneous but molecular clouds are the most structured with a hierarchy that is variously described as clumps and cores [7]. Clumps range from sizes $L \approx 0.5-5 \mathrm{pc}$ and masses $M \approx 10-10^{3} M_{\odot}$ and may contain young groups, or clusters, of stars. Cores are the smallest identifiable structures that form individual stars and are approximately spherical with typical radii $R \approx 0.05 \mathrm{pc}$ and mass $M \approx 1 M_{\odot}[8]$.

Figure 1 illustrates the hierarchy and huge range of spatial scales involved in the full star formation process from diffuse atomic gas to giant molecular cloud to cluster forming clump to individual star forming core.

The velocity dispersion of molecular clouds, $\sigma \approx 3-10 \mathrm{~km} \mathrm{~s}^{-1}$, is much larger than the sound speed, $\sigma_{\text {thermal }}=\left(k T / m_{\mathrm{H}_{2}}\right)^{1 / 2}=0.1 \mathrm{~km} \mathrm{~s}^{-1}$ where $m_{\mathrm{H}_{2}}$ is the mass of a hydrogen molecule, and is likely due to waves along magnetic field lines. Consequently, unlike the ionised and atomic 

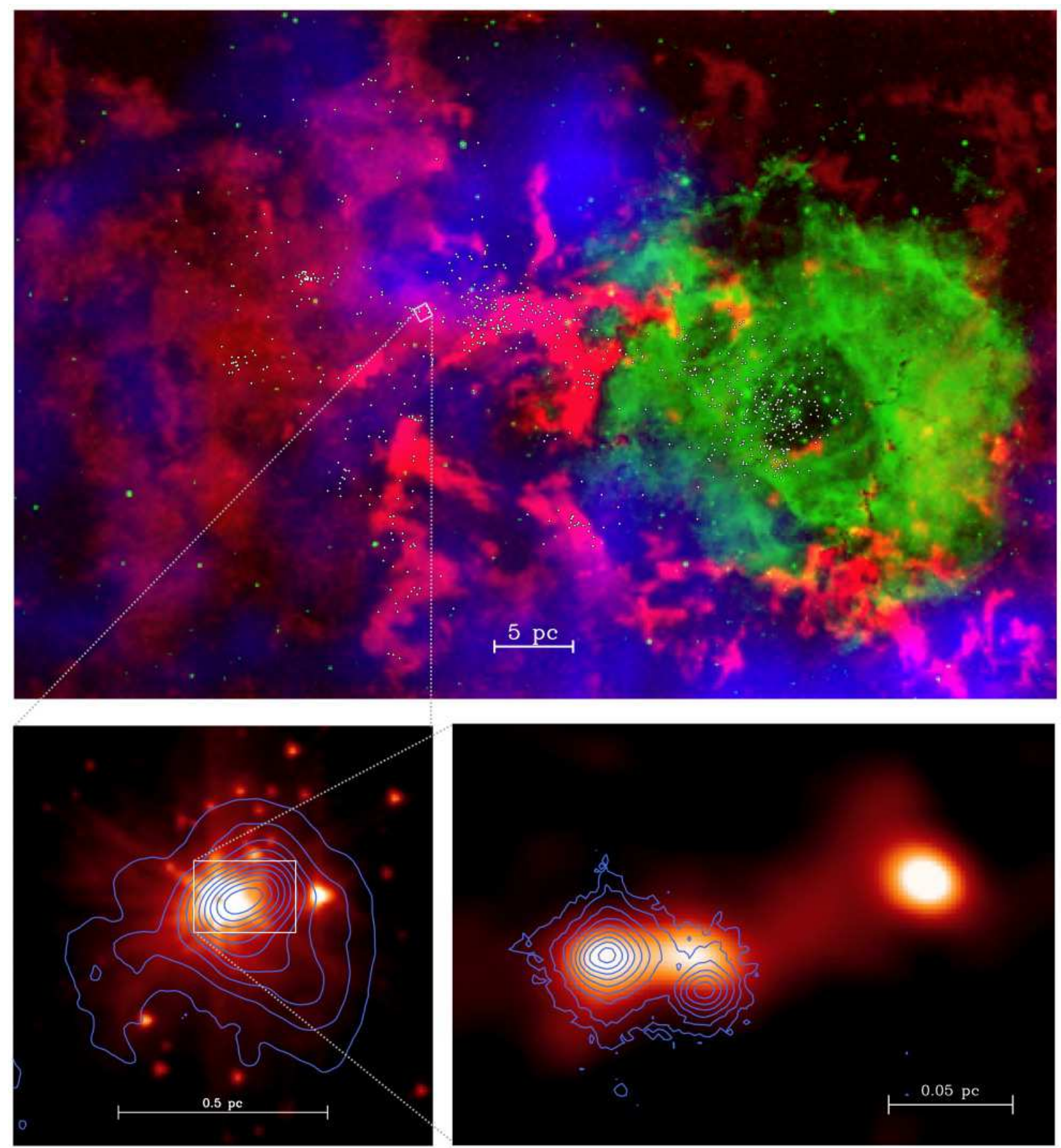

Figure 1. The scales of star formation. The upper panel shows a composite view of the Rosette nebula and accompanying cloud. The nebula, shown in green from a Digitized Sky Survey image, is powered by a collection of massive stars at the centre of a large cluster. The red image is $2.6 \mathrm{~mm}$ emission from the CO molecule and indicates the presence of a Giant Molecular Cloud, about $50 \mathrm{pc}$ in diameter and mass $2 \times 10^{5} M_{\odot}[9]$. The blue image shows the intensity of the neutral hydrogen $21 \mathrm{~cm}$ line, and reveals an atomic envelope around the cloud [10], either the remnants of the diffuse gas from which the molecular cloud formed or photodissociated gas due to the surrounding ultraviolet radiation field. The lower panels zoom in on the star formation process. The left panel shows contours of $850 \mu \mathrm{m}$ emission from a cold dusty envelope around a deeply embedded cluster, imaged at $3.6 \mu \mathrm{m}$. The right panel shows $4.9 \mu \mathrm{m}$ contours from embedded protostars on a $1.2 \mathrm{~mm}$ interferometric image of 3 dusty cores lying in a filament at the centre of the clump [11]. The scale bars decrease by an order of magnitude in each panel demonstrating the enormous change in scale from cloud to clump to core.

components of the interstellar medium, molecular clouds are not in pressure equilibrium with their surroundings. Either they are bound by their own self-gravity or they are transient features rather like the froth on an ocean wave. This is a case where the lack of precise measurements hinders our ability to differentiate between these two possibilities and it is hard to determine the lifetime of molecular clouds (or even tell the difference between young and old objects [12]). Current estimates, based on theories of cloud formation and destruction, range from $\approx 3-10 \mathrm{Myr}$ 
[13, 14]. The evolutionary state of the denser subunits, clumps and cores, within star forming clouds are easier to characterize and several lines of evidence suggest that stars form within a few gravitational free-fall timescales,

$$
t_{\mathrm{ff}}=\left(\frac{3 \pi}{32 G \rho}\right)^{1 / 2}
$$

where $\rho$ is the mass density [15]. For a number density $n_{\mathrm{H}_{2}} \approx 10^{10} \mathrm{~m}^{-3}, t_{f f} \approx 10^{5} \mathrm{yr}$

\section{$2.2 \quad$ Protostars and protoclusters}

As the magnetic or turbulent support of a dense molecular core is lost, gravitational collapse ensues. The release of gravitational energy produces a hot central object that is observable at infrared wavelengths. The onset of hydrogen fusion at a core temperature of $6 \times 10^{6} \mathrm{~K}$, signals the birth of of a protostar. The radiation and winds from the young star sweep away any remaining molecular material and it becomes optically visible [16].

Ground-based astronomy can detect protostars at $\lambda \lesssim 20 \mu \mathrm{m}$ but space-based instruments are required to observe cooler and fainter objects at longer wavelengths. The Spitzer and Herschel telescopes are now providing detailed views of the youngest protostars in molecular clouds. Infrared surveys of molecular clouds show that most stars do not form singly but in clusters ranging from 10 to $10^{6}$ members [17].

The number of clusters, $N_{\mathrm{c}}$ with $N_{*}$ stars follows a differential equation of the form [17, 18].

$$
\frac{d N_{\mathrm{c}}}{d N_{*}} \propto N_{*}^{-2}
$$

which implies that the probability that any given star forms in a cluster with $N_{*}$ stars is $P\left(N_{*}\right)=$ $\int N_{*} d N_{\mathrm{c}} \propto \log N_{*}$. That is, a star is as likely to be born in a rare, but large cluster with between $10^{5}$ and $10^{6}$ stars as in a far more common smaller group with between 10 and 100 stars. Even though our Sun is an isolated system today, it most likely formed with many, likely thousands of, siblings that have since dispersed during their twenty-plus orbits around the Galaxy.

Stars are born with a range of masses, $M \approx 0.1-100 M_{\odot}$, with a distribution that appears to be remarkably uniform [19]. The number of stars with mass $M_{*} \gtrsim 0.5 M_{\odot}$ follows a power-law distribution,

$$
\frac{d N_{*}}{d M_{*}} \propto M_{*}^{-2.35}
$$

Only a small fraction, $\approx 0.3 \%$, are massive enough, $M_{*}>8 M_{\odot}$, to become core-collapse supernova. The distribution is heavily skewed toward the low end in terms of numbers but the few massive stars dominate the luminosity and their radiation, winds, and eventual supernovae affect the interstellar medium thermally, dynamically, and chemically.

\subsection{Protoplanetary disks}

The huge compression from core to stellar scales $\left(\approx 10^{15} \rightarrow 10^{9} \mathrm{~m}\right)$ magnifies any initial spin in the cloud and results in a rotationally supported disk around a protostar. These disks initially funnel material onto the growing star but they are also the sites of planet formation, being dense enough for dust grains to aggregate and, in their midplane, cool enough for gaseous compounds such as water to freeze out.

Disks are warm near their central star and cool far away, $T \approx 10^{3}-10 \mathrm{~K}$. Consequently they radiate from infrared to millimeter wavelengths. Infrared surveys are the most sensitive 
indicators of the presence of a disk and large surveys of clusters with different ages show that the fraction of stars with disks decreases from an initial value close to unity to zero by by 6 Myr, and that the median disk lifetime is about $3 \mathrm{Myr}[20,21]$. Masses can be measured at millimeter wavelengths where the entire disk emits. Observations show disk masses ranging from $10^{-4}$ to $10^{-1} M_{\odot}$, around stars with ages $\lesssim 1 \mathrm{Myr}$. This brackets the mass required to form the planets in the Solar System, estimated to be $0.01 M_{\odot}[22,23]$. However, the millimeter emission decreases precipitously by $3 \mathrm{Myr}$ [24]. indicating that the emitting surface area has decreased, either due to disk dispersal or grain growth into relatively large (centimeter or greater) sized planetesimals.

Disks are small with initial radii about 100 times the Earth-Sun distance, $1 \mathrm{AU}=1.5 \times$ $10^{11} \mathrm{~m} \approx 5 \times 10^{-6} \mathrm{pc}$. They can only be imaged at millimeter wavelengths using the technique of interferometry, that of correlating the signals from multiple antennae. In the case of Orion, however, disks are seen silhouetted against the bright backdrop of the nebula in optical light. The Hubble Space Telescope images of these so-called proplyds are some of the most spectacular images of protoplanetary disks [25, 26]. About $15 \%$ of disks in young star forming regions have the combination of sufficient mass $M \geq 0.01 M_{\odot}$ within $R \leq 50$ AU to form a planetary system on the same scale as our own [27].

\section{Meteoritics}

\subsection{Chondrites}

Meteorites provide a unique way to infer the conditions of the early solar system in far greater detail than astronomical measurements of a distant protoplanetary disk. These rocks are the building blocks of planets that, until they landed on Earth, were not incorporated into a large solar system body. As such they are fossils of the early stages of planet formation. Using scanning electron microscopes and ion mass spectrometry cosmochemists can measure the structure, mineralogy and isotopic composition of meteorites to learn about the precise conditions of the environment in which they formed.

Meteorites can be stony, iron, or a mixture of the two. The most primitive are stony with a composition similar to that of the Sun. These so-called chondrites are themselves largely composed of chondrules, approximately spherical globules generally less than a millimeter across, made largely of silicates and small amounts of metals. Carbonaceous chondrites are a subclass with large abundances of refractory elements, those that condensed out of the protosolar nebula at relatively high temperatures, and therefore early in its history. In particular, carbonaceous chondrites contain many Calcium-Aluminium-rich inclusions, the oldest known solids in the early solar system with an age derived from radioactive lead isotopes to be $4567.2 \pm 0.6 \mathrm{Myr}$ [28].

Radioactive isotopes with much shorter half-lives show the time sequence of important events in the growth of planets and can be directly compared to astronomical observations of Myr-age protoplanetary disks.

\subsection{Short lived radionuclides}

An element can have unstable isotopes that are chemically identical but decay over cosmic timescales. A chondrule with a particular mineralogy can therefore be incorporated into a chondrite but subsequently change its composition. These isotopic anomalies are frozen into the material and, unless the chondrite undergoes further processing, are immutable. Short lived radionuclides have half-lives, $t_{1 / 2}$, comparable to planet forming timescales, variously defined as $10-100 \mathrm{Myr}$ [29]. and provide a natural chronometer for the growth of planets.

The first short lived radionuclide, ${ }^{129} \mathrm{I}$, with a mean life $\left(\tau=t_{1 / 2} / \ln 2\right)$ of $23.5 \mathrm{Myr}$ was discovered in meteorites almost 50 years ago [30]. Many others have since been detected [31]. Their importance lies in showing the timescales over which the products of stellar nucleosynthesis are transported through the interstellar medium to planetesimals. The total number of atoms 
of a radionuclide depends on a balance between production rate, $P$, from stellar nucleosynthesis and radioactive decay [32],

$$
\frac{d N}{d t}=P-\frac{N}{\tau}
$$

For a stable isotope, $\tau$ is effectively infinity, and $N=\int P d t$, the production rate integrated over the age of the Galaxy. For a finite mean life, the general solution to the above is

$$
N=\int_{0}^{t} P\left(t^{\prime}\right) e^{-\left(t-t^{\prime}\right) / \tau} d t^{\prime}
$$

The exponential within the integral strongly weights the number toward the recent production history (i.e., within a few mean lives). The predicted ratio of an short lived radionuclides relative to its stable partner in a chondrule must take into account the additional decay during the transport from hot, rarefied stellar ejecta to solid. Measured values are roughly consistent with a timescale of about $100 \mathrm{Myr}$ but there is a significant discrepancy from one element to another that may be attributable to different production rate histories [33, 34].

A few very short-lived short lived radionuclides with $\tau<3$ Myr stand out as having abnormally high abundances. An important example is ${ }^{26} \mathrm{Al}$, which decays into ${ }^{26} \mathrm{Mg}$ with a mean life $\tau=1 \mathrm{Myr} .{ }^{26} \mathrm{Mg}$ is stable and found in Calcium-Aluminium inclusions with an abundance that correlates with ${ }^{27} \mathrm{Al}$. The implication is that some of the ${ }^{26} \mathrm{Mg}$ results from the decay of ${ }^{26} \mathrm{Al}$. The situation is shown schematically in Figure 2. The extrapolation to zero aluminium content shows the baseline value of ${ }^{26} \mathrm{Mg}$ and the slope shows the contribution due to ${ }^{26} \mathrm{Al}$ decay. The inferred isotopic ratio in the early solar system, ${ }^{26} \mathrm{Al} /{ }^{27} \mathrm{Al}=5 \times 10^{-5}$, is more than an order of magnitude greater than the predicted steady state Galactic background [35].

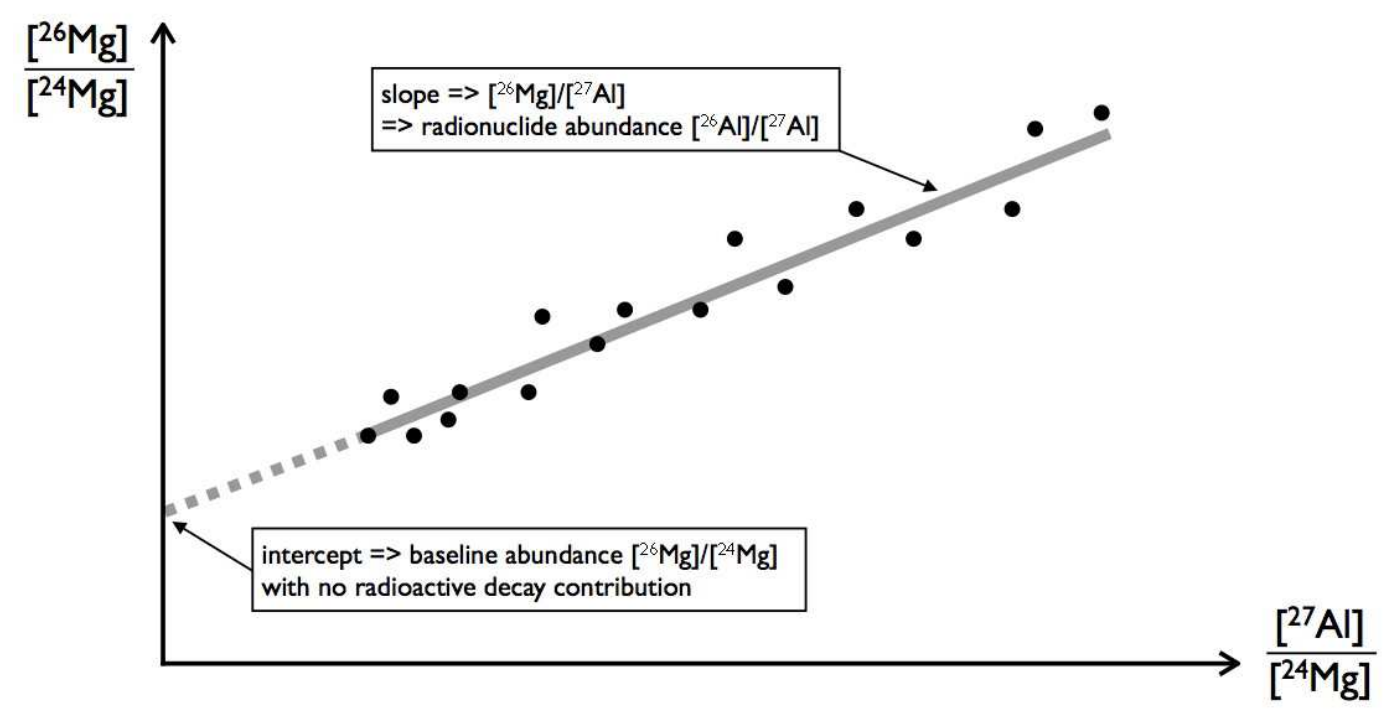

Figure 2. Schematic of the determination of the ${ }^{26} \mathrm{Al}$ isotopic abundance in Calcium-Aluminium inclusions.

The ${ }^{26} \mathrm{Al}$ Galactic background can be measured directly via $\gamma$-ray spectroscopy of its decay [36]. The resolution of these instruments is too low to show much detail although the Galactic rotation can be discerned showing that the emission is indeed coming from throughout the Galaxy. Compared with these measurements the inferred abundance of ${ }^{26} \mathrm{Al}$ in the early solar system is a factor of 6 above the Galactic background. The inescapable conclusion is that there was a significant spike in the local production rate of ${ }^{26} \mathrm{Al}$ slightly before or during the formation of the solar system. 
The discoveries of short-lived radionuclides in chrondrites not only provide important constraints to the solar birthplace environment but also have wider ramifications. The rapid decay and high abundance of ${ }^{26} \mathrm{Al}$ produces enough heat to melt and differentiate planetesimals at early times [37]. Such radiogenic heating may significantly move the snow line, the disk radius at which water freezes, outwards in a forming planetary system [38]. Thus the issue of the commonality of the environment of the early solar system, is of great import for understanding the water content of exoplanets and even their capacity to host technological civilizations [39]. Too little water would be inhospitable to life but too much water, covering all the land, would prohibit technological development. Although astronomers are only just at the cusp of being able to determine the composition of exoplanets, one ocean-world has now been convincingly demonstrated from radial velocity measurements of its mass and transit measurements of its radius [40]. Such density determinations provide a direct link between astronomical and cosmochemical studies.

\subsection{An external influence on the early solar system}

Two explanations were proposed to explain the high abundance of ${ }^{26} \mathrm{Al}$. An external enrichment of the protosolar nebula by the winds or supernovae of nearby massive stars [41] or a local production by the irradiation of chondrites by energetic particles from an active protosun [42].

External enrichment requires a synchronicity both in time, due to radioactive decay, and space, due to geometric dilution of the stellar ejecta. This led to the idea that, in some way, the death of a massive star somehow induced the birth of the solar system through, perhaps, the compression and subsequent collapse of a molecular core.

As our understanding of star formation increased, it was recognised that young protostars possess very strong magnetic fields and exhibit strong X-ray flares. The flares are due to magnetic reconnection events on the stelar surface and shoot particles (mostly hydrogen and helium nuclei) away at high energies. The same phenomenon occurs in solar flares today but at a much reduced frequency and at lower energies due to the much weaker present-day magnetic field. As dust grains accreted through the protosolar disk, they would have been exposed to a high particle flux and sufficient nuclear reactions would occur to explain many of the enhanced short lived radionuclides abundances. This picture is appealing in many ways, not the least of which is that it is self-contained and does not require any special circumstances for the early solar system.

A dichotomy has arisen in the literature as both external and internal mechanisms have considerable flexibility and can be adjusted to match the observed isotope abundances in chondrules. They need not be not mutually exclusive, however, and it appears that both mechanisms are needed to explain the observations. Stellar nucleosynthesis is unable to produce ${ }^{10} \mathrm{Be}$ at the levels observed in chondrules [43]. Its presence is definitive proof that the protosun did mutate some of the elements in the disk. On the other hand, protostellar particle fluxes are several orders of magnitude too low to match the observed abundance of the neutron-rich short lived radionuclides, ${ }^{60} \mathrm{Fe}[42]$.

${ }^{60} \mathrm{Fe}$ decays to ${ }^{60} \mathrm{Ni}$ with a mean life of $3.8 \mathrm{Myr}$ [44] and its presence in chrondrites was inferred by the correlation of the isotope ratio, ${ }^{60} \mathrm{Ni} /{ }^{61} \mathrm{Ni}$, with the main isotope of iron, ${ }^{56} \mathrm{Fe} /{ }^{61} \mathrm{Ni}$ [45]. Although the evidence for ${ }^{60} \mathrm{Fe}$ in chondrites does not appear to be in dispute, its precise abundance in the early solar system is not yet firmly established with values ranging from $\left[{ }^{60} \mathrm{Fe}\right] /\left[{ }^{56} \mathrm{Fe}\right]<10^{-7}$ [46] to $10^{-6}[47]$. Here, I use an intermediate value, $\left[{ }^{60} \mathrm{Fe}\right] /\left[{ }^{56} \mathrm{Fe}\right]=3 \times 10^{-7}$ [48] which places it about an order of magnitude greater than the model background level and a factor of 6 above direct $\gamma$-ray measurements [49].

The elevated abundance of ${ }^{60} \mathrm{Fe}$ is a clear and unambiguous signature of external enrichment of the early solar system. Understanding its transport from the core of a massive star to planetesimal in at most a few mean lives provides the strongest constraint on the astrophysical environment of the solar birthplace. In the following section I describe various short lived radionuclides enrichment scenarios. 


\section{External enrichment scenarios}

Iron and its short lived isotope ${ }^{60} \mathrm{Fe}$ can only be produced in the cores of massive stars. It can then be expelled into the interstellar medium either through stellar winds during the very late stages of their evolution as core material is convected up to the surface or during the death of a star as a core-collapse (Type 1b, 1c, or II) supernova. The fundamental issue is how to convert hot and rarefied stellar winds or supernova ejecta into dust grains in a cold, dense protoplanetary disk within a sufficiently short time that the radionuclide does not decay away. Both sources, stellar winds and supernovae, have historically been considered in the context of the ${ }^{26} \mathrm{Al}$ excess. I discuss each below but now with the requirement to match the cosmochemically inferred early solar system ${ }^{60} \mathrm{Fe}$ abundance.

\subsection{Winds from evolved stars}

The end state of a star begins when all the hydrogen in its centre is used up. The core then contracts under its own gravity, heats up, and shells of hydrogen and helium undergo fusion around it. The outer layers of the star expand due to the increased energy production and it becomes a red giant. The large size implies low surface gravity and substantial gas can escape in the form of a wind. Convective mixing from core to surface then provides a path for newly synthesized material to rapidly enter the interstellar medium. This mechanism is a potential explanation of the high abundances of many of the short lived radionuclides inferred in the early solar system [50]. Figure 3 shows a wind-blown bubble from an evolved star in a star-forming molecular cloud; a simultaneous demonstration that this situation can occur but that its effect is limited to a very small region of the cloud.

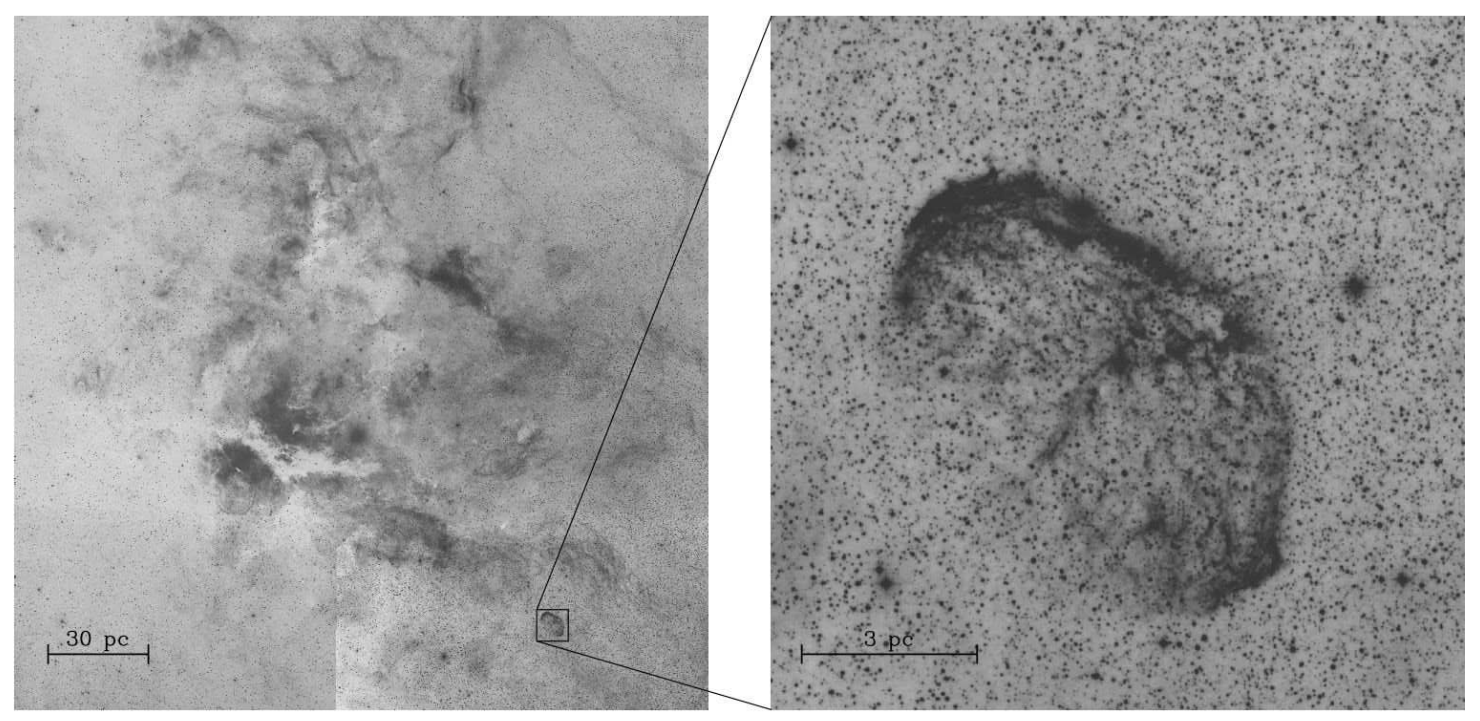

Figure 3. The relative scale of stellar wind ejecta compared to molecular clouds. The two panels are from the Digitized Sky Survey and show optical extinction and nebulosity associated with the IC1318 star forming region in the left panel. The right panel shows a close-up of the wind-blown bubble from the evolved star, HD 192163. Note the relative scales that graphically demonstrates how the pollution from a stellar wind is limited to only a small part of a molecular cloud.

The production of ${ }^{60} \mathrm{Fe}$ requires high core densities that are only realised in moderately massive stars with masses $M_{*} \gtrsim 5 M_{\odot}[51]$. It takes about $110 \mathrm{Myr}$ for a $5 M_{\odot}$ star to enter the red giant phase [52], much longer than molecular cloud lifetimes (2.1). More massive stars have shorter lifetimes but only the most massive, $M_{*} \gtrsim 20 M_{\odot}$, use up their core hydrogen within the $10 \mathrm{Myr}$ upper bound to giant molecular cloud lifetimes and they become supernovae shortly thereafter. The ${ }^{60} \mathrm{Fe}$ yield in supernovae dominates in this case, as discussed in subsequent subsections. 
The external enrichment of a planetary system by a red giant therefore requires the chance coincidence of a relatively old evolved star running into a molecular cloud just as it begins to form stars. Based on a census of known red giants and molecular clouds within $1000 \mathrm{pc}$ of the sun the probability of such serendipity is estimated to be $\approx 1 \%$ per Myr [53]. Further, the ratio of stellar wind to molecular mass is very small, $3 \times 10^{-4}$, so the likelihood that the first few Myr of the nascent solar system were enriched with ${ }^{26} \mathrm{Al}$ at the cosmochemically inferred levels is about $0.001 \%$. The same reasoning applies for the enrichment of ${ }^{60} \mathrm{Fe}$ but with an even lower likelihood due to the required high stellar masses.

Catalogs of evolved stars with high mass loss rates extend beyond the solar neighborhood [54] and can be extrapolated to make a more general calculation for the entire Galaxy. Their average surface density is about $10^{-5} \mathrm{pc}^{-2}$, independent of Galactic radius. This implies a total population of about $3 \times 10^{3}$ in the Galaxy. Each object remains in this phase for about $1 \mathrm{Myr}$ and ejects a total of about $3 M_{\odot}$ into the interstellar medium. Modeling the isotopic abundances in the winds, each stars can pollute about 100 times more mass to early solar system levels or $\approx 10^{6} M_{\odot}$ in total. The total molecular mass in the Galaxy is $1 \times 10^{9} M_{\odot}[55$ ] so the proportion of star forming material that can be enriched is no more than $0.1 \%$. This assumes that the star formation efficiency is independent of the presence of an evolved star which is entirely consistent with observations. Note that this is a generous upper limit since it does not include the low likelihood that an evolved star lie within or near a molecular cloud, nor does it include radioactive decay during the transport from stellar wind to planetesimal. Finally, again no account has been made to consider the subset of evolved stars the are massive enough to produce ${ }^{60} \mathrm{Fe}$. Even without these additional factors, however, this simple calculation provides a robust and independent demonstration that the pollution of the early solar system by the winds from an evolved star is an extremely unlikely scenario for the origin of ${ }^{60} \mathrm{Fe}$ in the early solar system.

\subsection{Supernovae}

The most massive stars, $M>8 M_{\odot}$, have such high central pressures that shell burning around a hydrogen depleted core progresses to more neutron rich elements up to iron, at which point nuclear fusion is no longer an exothermic process. Without a source of thermal pressure to counteract self-gravity, the star collapses in on itself. The release of gravitational potential energy produces a supernova explosion and many of the freshly synthesized elements are ejected and can be recycled into a new generation of stars and planets. Indeed, almost all the iron on Earth was produced in supernovae but the presence of its isotope, ${ }^{60} \mathrm{Fe}$ with mean life $\tau=3.8 \mathrm{Myr}$, ties the death of a massive star to the nearly simultaneous formation of the solar system.

Compared to stellar winds, supernovae are an instantaneous event but the huge energy release impacts a much larger volume. The initial speed of supernovae ejecta is $\approx 10^{4} \mathrm{~km} \mathrm{~s}^{-1}$, and supernovae remnants can expand to hundreds of parsecs in diameter [56]. Their influence on the different scales of star and planet formation, however, depends on a number of factors which is incorporated into the concept of radioactivity distance.

\subsubsection{The radioactivity distance}

In the transport of an short lived radionuclide from supernova to planetesimal, there will be losses due to radioactive decay, geometric dilution, and efficiency of incorporation into solids. Assuming that a mass, $M_{\mathrm{SLR}}$, is ejected isotropically, the resulting mass fraction in a target at distance $D$ with mass $M_{\mathrm{t}}$ amd projected area $A_{\mathrm{t}}$ is,

$$
X_{\mathrm{SLR}}=f_{\mathrm{inj}} \frac{M_{\mathrm{SLR}}}{M_{\mathrm{t}}} \frac{A_{\mathrm{t}}}{4 \pi D^{2}} e^{-t / \tau}
$$

where $f_{\text {inj }}$ is the injection efficiency [57].

Relative to hydrogen, the solar iron abundance is $\left[{ }^{56} \mathrm{Fe}\right] /[\mathrm{H}]=3.45 \times 10^{-5}$ so the chondritic isotopic ratio $\left[{ }^{60} \mathrm{Fe}\right] /\left[{ }^{56} \mathrm{Fe}\right]=3 \times 10^{-7}$ corresponds to a mass fraction $X_{60 \mathrm{Fe}}=4.4 \times 10^{-10}$ (the 
product of the two abundances times the ratio of the mass of ${ }^{60} \mathrm{Fe}$ to the mean atomic mass $1.4 m_{\text {proton }}$ from an admixture of $90 \%$ hydrogen and $10 \%$ helium). This is the number that must be matched or exceeded by any transport scenario.

Stellar nucleosynthesis models yield $M_{60 \mathrm{Fe}}=2 \times 10^{-6}-1 \times 10^{-3} M_{\odot}$ for supernova progenitor masses $M_{*}=11-120 M_{\odot}$ [58]1]. Taking the stellar mass function weighted mean, $M_{60 \mathrm{Fe}}=$ $1.1 \times 10^{-5} M_{\odot}$, and assuming a maximum $f_{\text {inj }}=1$ and negligible transport time, $t \ll \tau$, gives an upper limit to the distance between a source and target for enrichment of ${ }^{60} \mathrm{Fe}$ to early solar system levels,

$$
D_{60}(\mathrm{pc})=45 \Sigma_{\mathrm{t}}^{-1 / 2}\left(\mathrm{M}_{\odot} \mathrm{pc}^{-2}\right)
$$

where $\Sigma_{\mathrm{t}}=M_{\mathrm{t}} / A_{\mathrm{t}}$ is the projected surface density of the target. This radioactivity distance serves as a rough guide to the relevant scales of interest. Four different enrichment scenarios are ordered below by target scale from smallest (disks) to largest (giant molecular clouds) ending with what I believe to be the most probable solution, that of an enhanced background in spiral arms.
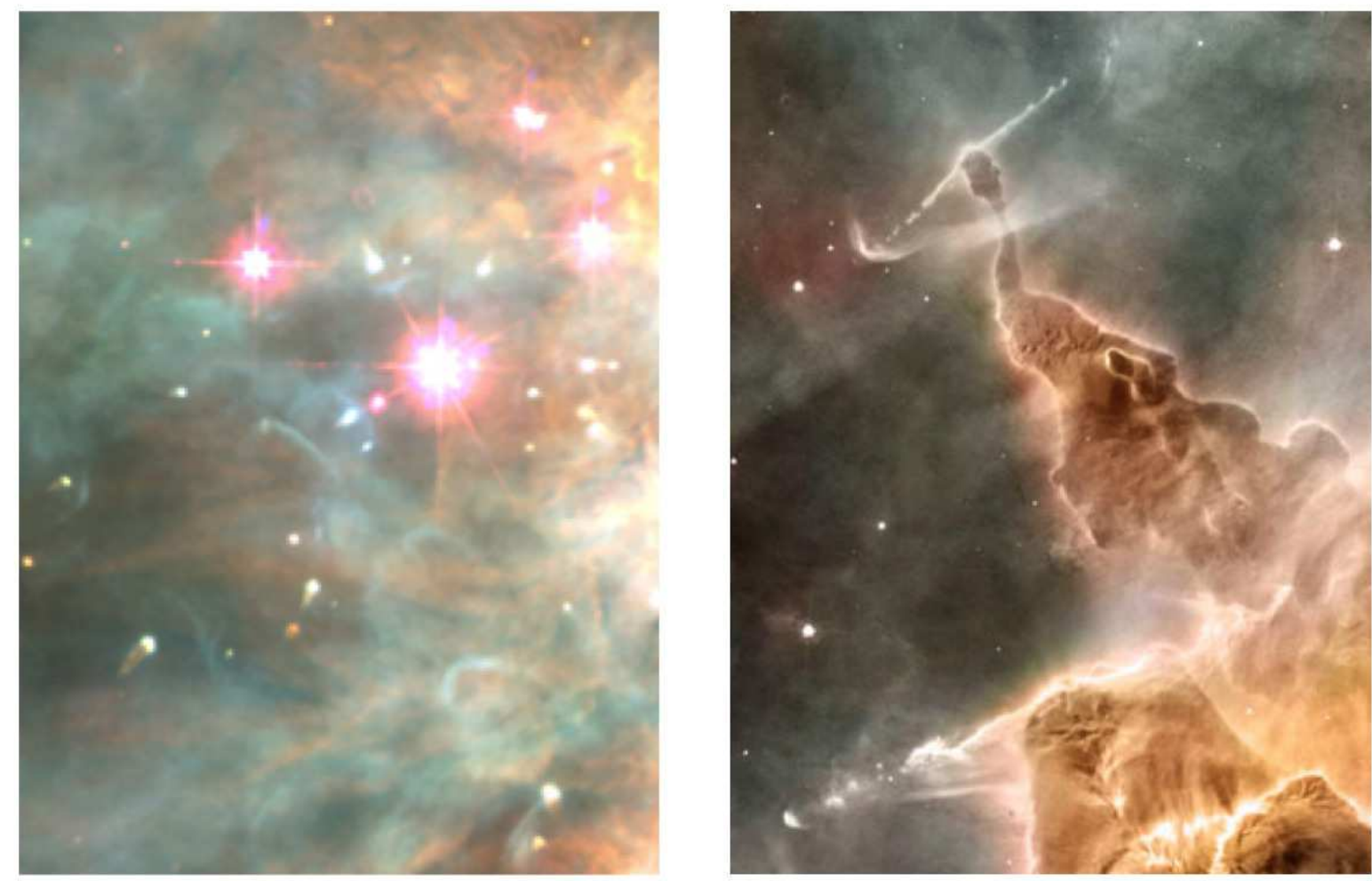

Figure 4. Hubble Space Telescope images of star and planet formation within or on the boundaries of ionized regions around massive stars. The bright stars in the left panel is the Trapezium Cluster in Orion. Cometary tails of photoevaporating protoplanetary disks around low mass stars can be seen pointing back toward the brightest star, $\theta_{1}$ Ori C. The right panel shows two star-forming pillars in the Carina nebula. Jets from young protostars at the tips of the pillars indicate very recent, potentially triggered, star formation.

\subsubsection{Injection into a protoplanetary disk}

Clusters extend in size up to about $N_{*} \approx 10^{6}$ stars so, with the cluster and number distributions as described in $₫ 2.2$, the median cluster size is $N_{*} \approx 10^{3}$ which is sufficient to contain a star

\footnotetext{
1 The mass fraction of ${ }^{60} \mathrm{Fe}$ relative to ${ }^{56} \mathrm{Fe}$ is about $10^{-5}$ in supernova ejecta, about two orders of magnitude greater than the ratio in primitive rocks so the overall iron abundance in the solar system is increased by only about $1 \%$ from the ${ }^{60} \mathrm{Fe}$ injection event, and would not be discernable in the galactic metallicity distribution.
} 
massive enough to become a supernova. Direct evidence that the solar system formed in a large cluster comes from the sharp edge to the radial distribution of Kuiper belt objects (rocky bodies beyond the orbit of Neptune) which has been attributed to the external photoevaporation of the protoplanetary disk by a nearby hot, ionizing star [59]. The Trapezium Cluster in Orion is the closest example of such a star forming environment (Figure 4). As the massive stars end their lives as supernovae, nearby protoplanetary disks could, in principle, be directly injected with ${ }^{60} \mathrm{Fe}$ [60].

For a disk with the minimum mass necessary to form the solar system, $M=0.01 M_{\odot}$, in a radius $R=50 \mathrm{AU}$, the face-on surface density, $\Sigma=5 \times 10^{4} M_{\odot} \mathrm{pc}^{-2}$ implies a radioactivity distance $D_{60}=0.2 \mathrm{pc}$. This is on the limit at which a protoplanetary disk can survive a supernova explosion [61] which immediately suggests that it is an unlikely scenario.

An additional problem is the timescale for massive stars to become supernovae compared to disk lifetimes. Even the most massive stars, $M_{*}=100 M_{\odot}$, take about $3 \mathrm{Myr}$ to burn their core hydrogen and begin nucleosynthesis of heavy elements at which point about half the disks in the cluster will have disappeared. Further, only stars with masses $M_{*}>30 M_{\odot}$ explode within $6 \mathrm{Myr}$, the maximum disk lifetime. Stars this massive are extremely rare and only found in very large clusters, $N_{*} \gtrsim 10^{4}$. The situation is graphically illustrated in Figure 5 ,

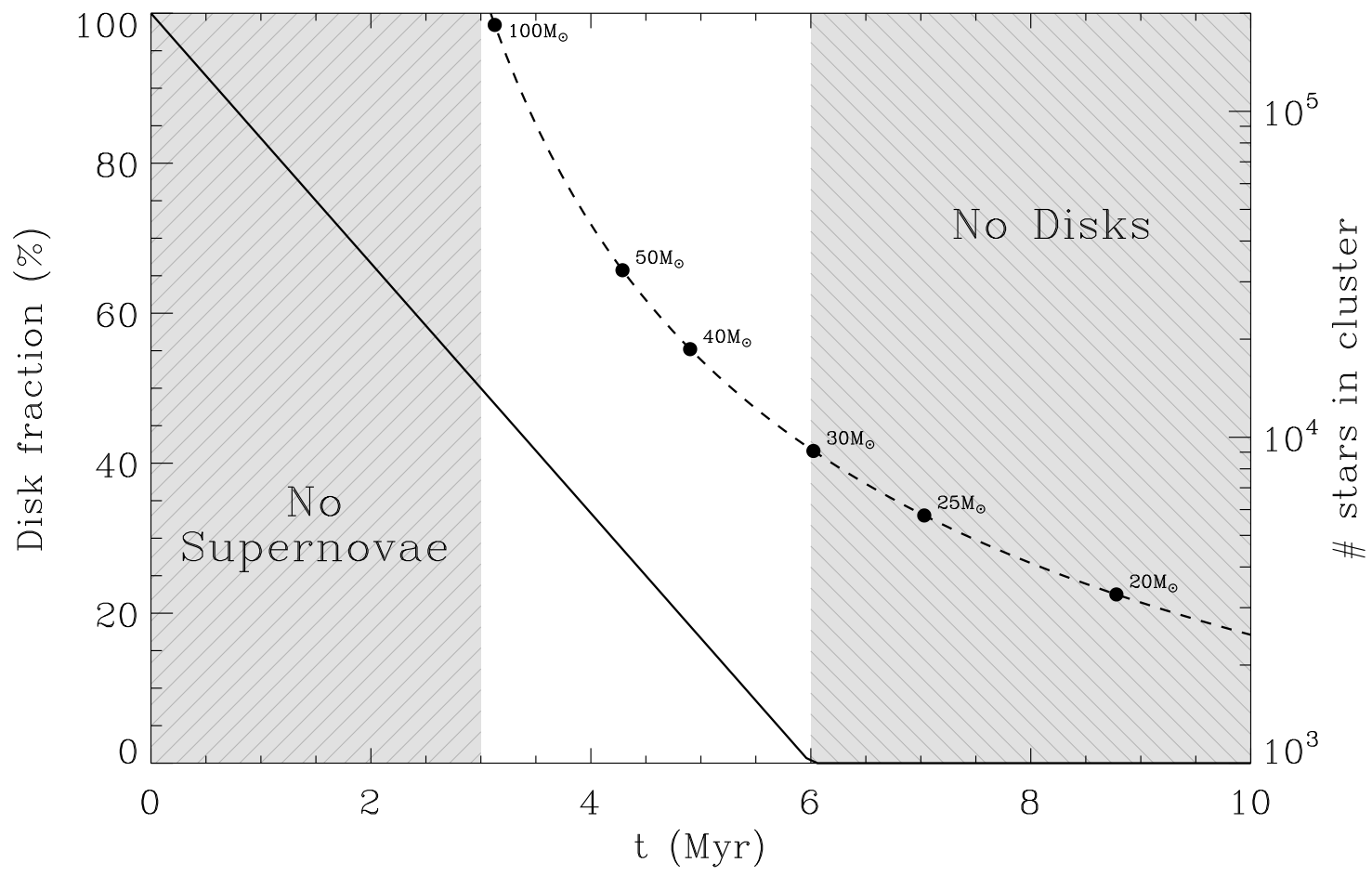

Figure 5. The lifetime problem for direct supernova injection into a protoplanetary disk. The solid diagonal line decreasing from $100 \%$ to $0 \%$ schematically represents the decrease in the disk fraction in clusters of varying age 20]. The curved dashed line plots the expected cluster size required to host a massive star with main sequence lifetimes from 3 to 10 Myr. Stars of different mass are labeled along this line from $20-100 M_{\odot}$. The hashed areas show that there are no supernovae before $3 \mathrm{Myr}$ and no disks remain after $6 \mathrm{Myr}$. This leaves only a small range in time, $3-6 \mathrm{Myr}$, when there are both disks and a supernova. Moreover, only stars with masses greater than $30 M_{\odot}$ satisfy this constraint and they are extremely rare, found only in clusters of $10^{4}$ stars or more.

The timescale problem is only slightly mitigated by allowing the massive star to form before the low mass stars since the main sequence lifetime increases sharply as the stellar mass decreases. For instance, the lifetime of a $20 M_{\odot}$ star is about $9 \mathrm{Myr}$ but this is still a relatively rare star, found only in clusters with $N_{*}>3000$, somewhat larger than Orion. For disks to survive at the 
time this becomes a supernova would require that it get a head-start of at least $3 \mathrm{Myr}$ in its evolution but there is no evidence that massive stars form that far before other stars in a cluster and, indeed, the situation appears to be quite the opposite [62].

Modeling cluster evolution and taking into account both the spatial and temporal constraints shows that no more than $1 \%$ of stars in an Orion-like cluster with $N_{*} \approx 10^{4}$ match the ${ }^{60} \mathrm{Fe}$ solar system abundance [63, 64]. The probability is lower in smaller clusters since they would be unlikely to host a supernova before all the disks disappeared and also lower in larger clusters since most disks would lie beyond the radioactivity distance. Allowing for multiple supernovae increases the odds and favors the largest clusters but, integrating over the full cluster distribution in equation 2, the probability that any given star in the Galaxy was enriched with ${ }^{60} \mathrm{Fe}$ by the direct injection of supernova ejecta into a disk is a statistically rare event, $<1 \%$.

\subsubsection{Triggered star formation}

Some stellar associations in molecular clouds consist of spatially and kinematically distinct subgroups ordered in age. The canonical example is the four Orion groups, OB1a-d, spaced sequentially from north to south and with ages from $\approx 10$ to $\approx 1$ Myr respectively [65]. Such arrangements led to the idea of triggered collapse of molecular gas on the boundary of an expanding ionised (H II) region [66]. Images of young stars on the brightly illuminated edges of an H II region abound in the literature in part because of their visual appeal, e.g., Figure 4, but the number of protostars is generally far less than those at the center of the H II region and it is unclear how representative this mode of star formation really is.

Nevertheless, if the formation of the Sun was indeed triggered, the protosolar nebula may have inherited the fresh products of nucleosynthesis from the massive stellar winds and subsequent supernovae [67]. There is much work on the observations and theory of triggered star formation [68] but there are two main mechanisms: the compression of a molecular cloud core to sufficiently high pressures that it becomes gravitationally unstable and undergoes collapse, and the sweeping up of gas to unstable densities and subsequent collapse.

A possible example of the former is the famous "pillars of creation" in the Eagle Nebula [69]. For a typical core with $M=1 M_{\odot}, R=0.05 \mathrm{pc}, \Sigma=130 M_{\odot} \mathrm{pc}^{-2}$, the radioactivity distance $D_{60}=4 \mathrm{pc}$. This can be refined by numerical models of supernova ejecta impacting a core [70] showing that $f_{\text {inj }} \approx 0.1$ which implies a reduced $D_{60}=1.2 \mathrm{pc}$. This is comparable to the distance from the ionizing star to the pillars in the Eagle nebula which is encouraging.

This picture is deceptive, however, in that the ionizing star is many Myr away from becoming a supernova. Well within that timeframe, the powerful stellar winds and photoionization will sweep away and ablate any cores [71, 72]. This is consistent with millimeter wavelength observations that show very little molecular gas toward the centre of ionised or wind-blown stellar bubbles [73, 74].

A final and fatal blow to this scenario has been struck with the most recent high resolution numerical simulations that show successful triggering models have very low injection efficiencies, $f_{\text {inj }} \approx 10^{-3}[75]$. This reduces $D_{60}=0.1 \mathrm{pc}$ which is so close to a supernova that the core would almost certainly be destroyed. Even if somehow, the ejecta were slowed by dense intervening gas before impacting the core, the volume argument that showed a low enrichment likelihood for supernova injection of disks in 4.2 .2 would apply to cores too.

Triggered star formation may also occur at scales larger than cores by sweeping up surrounding molecular material to high densities and subsequent collapse [76]. The winds and supernova ejecta from the previous generation of stars may then enrich any resulting planetary systems with some short lived radionuclides at levels comparable to those in the early solar system [77]. In this scenario, the relevant scales are those of clumps with typical masses $M \approx 10^{3} M_{\odot}$ and (irregularly shaped) sizes $L \approx 5$ pc. This implies surface densities, $\Sigma=M / L^{2} \approx 40 M_{\odot} \mathrm{pc}^{-2}$, and a radioactivity distance $D_{60} \approx 7 \mathrm{pc}$.

Relative to the pressure driven collapse of a dense core, however, the "collect-and-collapse" of a clump is slow and the delay between injection of supernova ejecta and star formation results in significant decay of ${ }^{60} \mathrm{Fe}$. The low density that makes clumps good targets for injection also means 
they have a long free-fall timescale. The volume density $\rho \approx M / L^{3} \approx 5 \times 10^{-22} \mathrm{~g} \mathrm{~cm}^{-3}$, which implies a free-fall timescale $t_{\mathrm{ff}} \approx 3 \mathrm{Myr}$. The decay term, $e^{-t_{\mathrm{ff}} / 2 \tau}$, therefore becomes significant. The radioactivity distance reduces to $D_{60} \approx 5 \mathrm{pc}$, which is smaller than the radii of mature H II regions, such as the Rosette nebula in Figure 1. The injection efficiency may be higher in lower density clumps than in cores or disks but it is still likely to be substantially less than unity, reducing $D_{60}$ still further.

Figure 6 illustrates the different regimes in a contour plot of radioactivity distance in $M-R$ space where we have now allowed for both mass and geometric dilution, $D_{60} \propto \Sigma^{-1 / 2}$, and radioactive decay during free-fall, $D_{60} \propto e^{-t_{\mathrm{ff}} / 2 \tau}$. The injection efficiency is incorporated as a universal scale on the absolute contour levels, $D_{60} \propto f_{\text {inj }}^{1 / 2}$, independent of $M$ and $R$.

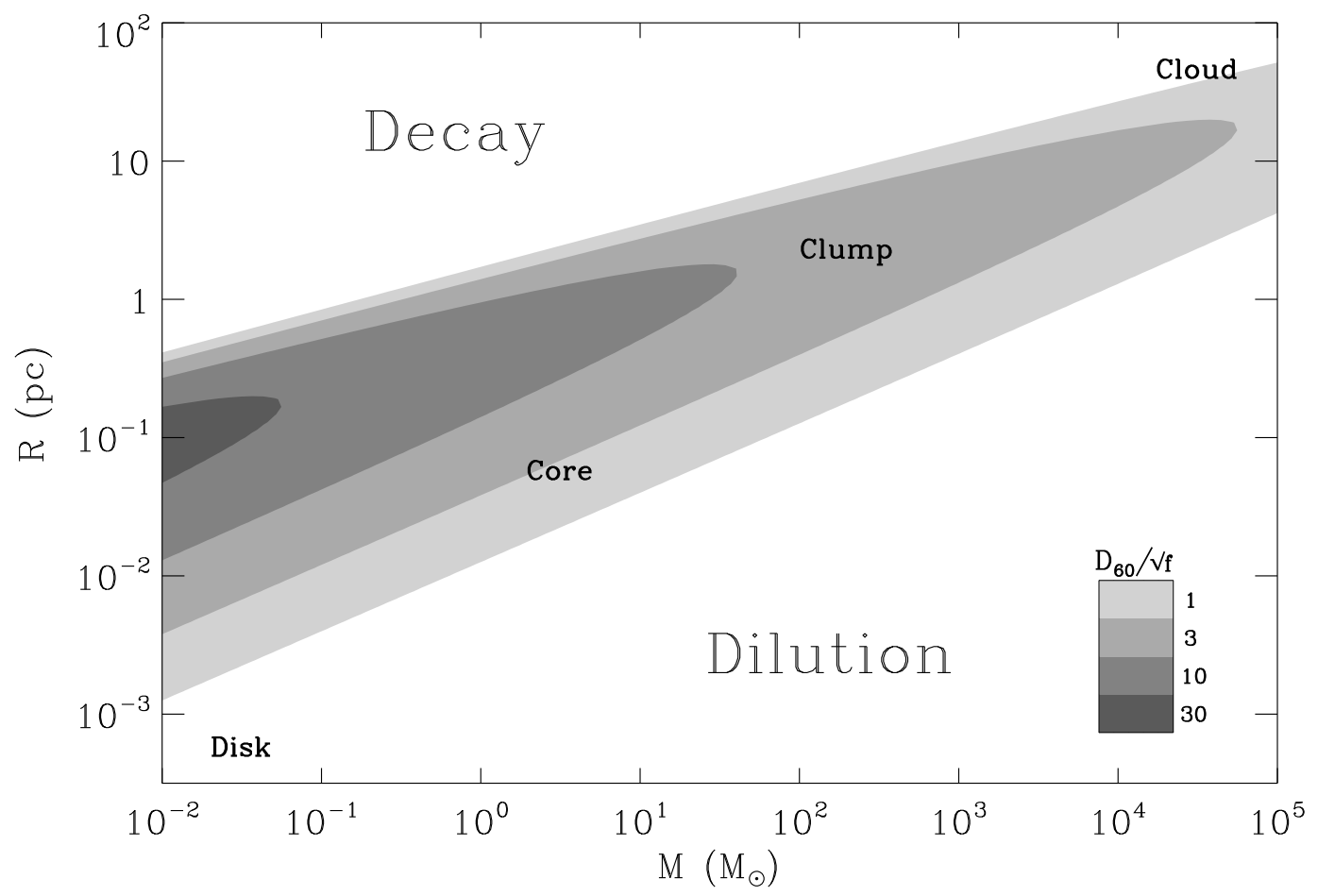

Figure 6. Contours of ${ }^{60} \mathrm{Fe}$ radioactivity distance as a function of the mass and radius of the enriched object. Objects with high surface densities cannot capture enough ejecta to match early solar system values. This region is labeled "Dilution". Objects with low volume densities have free-fall timescales much greater than the ${ }^{60} \mathrm{Fe}$ mean life. This region is labeled "Decay". The approximate location of the different regimes of star formation, from clouds to disks, are labeled. The injection efficiency is included as a scaling to the contour levels, $D_{60} \propto f_{\text {inj }}^{1 / 2}$.

This figure assumes spherical objects, i.e., $\Sigma=M / \pi R^{2}, \rho=3 M / 4 \pi R^{3}$. Different geometries produce only minor changes. Equation 6 also implicitly assumes the supernova ejecta are isotropically distributed. In fact, observations of supernova remnants show a great deal of inhomogeneity [78]. In principle, this allows enrichment of a smaller number of objects (disk, core or clump) at greater distances than $D_{60}$. The net effect is to decrease the chance of enrichment for any given object. Specifically, let the volume filling factor be $f_{v}$. Then the ejecta are spread over an area $4 \pi D^{2} f_{v}$ at distance $D$ from the supernova. Compared to the isotropic case, any object along this path can be enriched at a greater distance, $D=D_{60} f_{v}^{-1 / 2}$, but, on average, the number of objects that are affected is smaller by a factor $f_{v}$ because they have no preferred distribution relative to the supernova blast wave. The overall number of enriched objects is the product of these and therefore proportional to $f_{v}^{1 / 2}<1$. As a concrete example, consider the extreme case where all the ejecta are shot out in a narrow beam enriching one object very far 
from the supernova but leaving all other objects unaffected.

Figure 6 also shows that the decay problem is even worse at cloud scales where their low densities imply very slow evolution. The final mechanism bypasses this issue by effectively creating a long-lived but localised enriched background within which cloud and star formation take place.

\subsubsection{Supernova induced cloud formation}

With the growing realization that the conditions for ${ }^{60} \mathrm{Fe}$ enrichment are unlikely to be due to the direct injection of supernova ejecta into protoplanetary disks or protostellar cores, the possibility that supernova ejecta compress their surroundings and induce the formation of a molecular cloud was proposed [79]. Large clusters contain many massive stars that will, because of their different masses, produce multiple supernova at a cadence of every few Myr. This maintains the enrichment of the cloud as it forms a second generation of stars. This Supernova Propagation and Cloud Enrichment (SPACE) model has the advantage that short lived radionuclides are injected into relatively low density gas over many Myr and should therefore have a relatively high efficiency of incorporation into star and planet forming material. It is also appealing in that it can produce large numbers of ${ }^{60} \mathrm{Fe}$-rich systems over the entire cloud without any spatial preference. The conditions of the early solar system would then be expected to be commonplace.

A critical component of the model is the dynamic conversion of hot rarefied supernova ejecta into cool atomic and then molecular material via the collision of large scale shocks and flows in the interstellar medium [13]. If a large stellar association, $N_{*}=5000$, remains nearby over this time, about 10 supernova will occur over $20 \mathrm{Myr}$ and provide an approximately constant flux of ${ }^{60} \mathrm{Fe}$ into the cloud.

In fact the SPACE model may apply on larger scales and more generally due to the organization of molecular clouds and massive stars in the spiral arms of the Galaxy. Diffuse gas is compressed upon entry into a spiral density wave allowing, first molecules, and then stars to form. Almost all short-lived massive stars are born in spiral arms and many explode as supernova before they can migrate away [80]. This suggests that spiral arms are naturally enriched in short lived radionuclides, including ${ }^{60} \mathrm{Fe}$.

In classical spiral arm theory, molecular clouds form before the stars and tend to lie upstream of the spiral density wave [81]. Recent numerical simulations of the feedback from supernova on cloud formation and destruction and the maintenance of spiral arm structure show that the collision of supernova bubbles can form new clouds and propagate further star formation, depending on how much momentum is injected within the disk [82]. These would appear to be precisely the conditions required for the SPACE model but now on spiral arm scales.

Due to our location within the Galactic disk, it is hard to disentangle the projected view of the sky and decipher the relation between stars and clouds over spiral arm scales. However, we can detect giant molecular clouds and luminous clusters in nearby galaxies and make a plausibility test of this scenario for ${ }^{60} \mathrm{Fe}$ enrichment. Figure 7 plots the distribution of clouds and massive stars in the nearly face-on spiral M51 [83, 84]. The clusters and clouds follow the spiral arm pattern, of course, but there is clearly considerable overlap between young clusters, old clusters, and clouds as might be expected from a feedback between star and cloud formation. All the clusters shown here are large with $N_{*} \gtrsim 10^{3}$ and, assuming the same stellar mass distribution as in the Galaxy, would be expected to contain several supernova progenitors. Averaged over several Myr, this would produce a fairly constant flux of ejecta into the surrounding interstellar medium. To estimate the ${ }^{60} \mathrm{Fe}$ background, I calculate the mean mass of supernova ejecta from the luminosity of each cluster and spread it out as an inverse square law profile to a radius of $500 \mathrm{pc}$, the mean of these superstructures [56]. The rightmost panel plots the normalized background, averaged over the dashed box, enclosing the side of the galaxy where the cluster identification is complete and including a representative central region and both spiral arm and interarm regions. This simple model of the short lived radionuclides background is highly inhomogeneous with enhancements of up to a factor of 10 in the centre and some parts of the arm. Cross-correlating with the cloud catalog shows that $85 \%$ of the molecular gas mass is enriched above the average background level and $15 \%$ is enriched by more than a factor of 5 . The factor of 
6 enrichment in the ${ }^{26} \mathrm{Al}$ and ${ }^{60} \mathrm{Fe}$ levels of the early solar system relative to the observed $\gamma$-ray average background is therefore still high but not excessively so, with a percentage likelihood measured in the double rather than low single digits. This generalizing of the SPACE scenario to Spiral Arm Formation of Enriched clouds might be whimsically thought of as locking up the radionuclides in SAFE clouds.
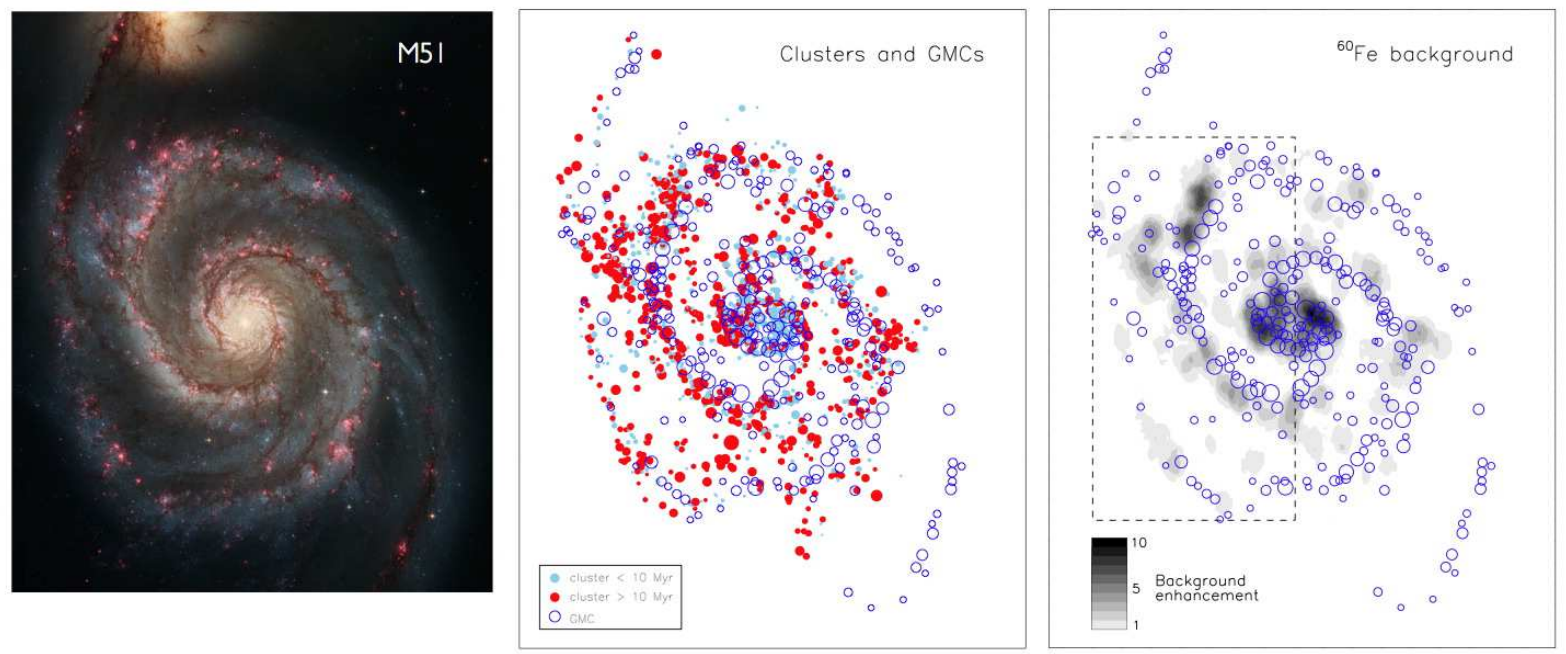

Figure 7. Spiral Arm Formation of Enriched (SAFE) clouds. The left panel shows a Hubble Space Telescope image of the M51 (Whirlpool) galaxy, a "grand design" spiral. The central panel shows the location of stellar clusters, colour coded by age [84] and cataloged molecular clouds with symbol size representative of mass [83]. The clusters overlap in age and are closely correlated with the location of the molecular clouds in the spiral arms. The right panel shows the molecular clouds overlaid on a simple model of the radionuclide background, scaled to the average within the dashed box.

\section{Summary}

Stars form in the densest cores of the densest components, molecular clouds, in the interstellar medium. Most stars form in large clusters with more than $10^{3}$ stars, and therefore likely in close proximity to several massive stars. The oldest identifiable pieces of meteorites show isotopic anomalies that indicate the presence of short lived radionuclides that were incorporated into planetesimals at very early times. Some may simply have been part of an approximately steadystate background resulting from a balance between production in massive stars and decay. Others may have been produced by nuclear reactions with energetic particles from an active early Sun. The high abundance of ${ }^{60} \mathrm{Fe}$, however, shows that the early solar system incorporated fresh nucleosynthetic products from massive stars.

The winds from evolved stars could impart some short lived radionuclides into a planetary region but require a highly unlikely chance encounter between an old star and young star forming region.

The massive stars that likely formed in the same cluster as the Sun are also highly unlikely to the be the source of this ${ }^{60} \mathrm{Fe}$. First, the stellar evolutionary timescales are relatively long compared to disk lifetimes. Second, and more importantly, protoplanetary disks and star-forming cores are small and must be very close to a massive star when it explodes as a supernova to capture the required amount of ejecta. Most objects would be much further away.

Star formation occurs in many sites within a molecular cloud and moderate mass clumps on the boundaries of H II regions can, in principle, capture a ${ }^{60} \mathrm{Fe}$ mass fraction comparable to that inferred in the early solar system. The low densities of these objects, however, imply long collapse times and there would be substantial decay before incorporation of the radionuclide into planetesimals. 
If we are to explain the ${ }^{60} \mathrm{Fe}$ levels in our early solar system as a not-uncommon event, we are forced to consider cloud scales and beyond. Large clusters produce multiple supernovae which result in an approximately steady local production of short lived radionuclides over about $20 \mathrm{Myr}$. Dense gas can result from the shocks at the interfaces of expanding supernovae ejecta and the enriched material may be rapidly processed into subsequent generations of stars. The conditions within spiral arms may be naturally conducive to this and I have presented a simple plausibility study using catalogs of known clouds and clusters in M51 to show that spiral arm enhancements of supernova ejecta may be up to an order of magnitude higher than the average. Many details remain to be filled in, not the least of which is an observational demonstration of cloud formation at bubble intersections.

I have focused here exclusively on matching the initial abundance of ${ }^{60} \mathrm{Fe}$. Supernovae imprint their signatures in other ways including the high solar system ${ }^{18} \mathrm{O} /{ }^{17} \mathrm{O}$ ratio [85]. However the link between the death of a massive star and our origins occurred, a full accounting needs also to be reconciled, as much as possible, with the full meteoritic record [86].

The presence of ${ }^{60} \mathrm{Fe}$ in chondrites presents a major challenge to theories of star and planet formation. It would be tempting to consider it simply as a fluke were it not for the possible anthropic implications [39]. If the delivery of ${ }^{60} \mathrm{Fe}$, and by extension other short lived radionuclides including ${ }^{26} \mathrm{Al}$, into our solar system were a rare exception, the radiogenic baking of the planetesimals in the early solar system would be much higher and the resulting water content of the planets lower then the norm. The first "ocean planet" has now been discovered [40], and as the statistics on exoplanet composition build up we will be able to place the water content of the Earth in context. Understanding the nature of our birth environment and the consequences thereof, is an important part of this picture.

\section{Acknowledgements}

I thank Sasha Krot, Gary Huss, and Eric Gaidos for introducing me to the puzzle of ${ }^{60} \mathrm{Fe}$ and the referees for their helpful comments which improved the manuscript. My work in this area has benefited greatly from discussions with Thierry Montmerle, Matthieu Gounelle, Ed Young, and Jeff Hester. Support for my research comes from the NSF and NASA.

\section{References}

[1] C.F. McKee and E.C. Ostriker, Theory of Star Formation, ARA\&A 45 (2007), pp. 565-687.

[2] A. Chrysostomou and P.W. Lucas, The Formation of Stars, Contemporary Physics 46 (2005), pp. 29-40.

[3] A.N. Halliday, Radioactivity, the discovery of time and the earliest history of the Earth, Contemporary Physics 38 (1997), pp. 103-114.

[4] F.C. Adams, The Birth Environment of the Solar System, ArXiv e-prints (2010).

[5] A.G.G.M. Tielens The Physics and Chemistry of the Interstellar Medium, , 2005.

[6] D.P. Cox, The Three-Phase Interstellar Medium Revisited, ARA\&A 43 (2005), pp. 337-385.

[7] J.P. Williams, L. Blitz, and C.F. McKee, The Structure and Evolution of Molecular Clouds: from Clumps to Cores to the IMF, Protostars and Planets IV (2000), pp. 97-+.

[8] J. di Francesco, N.J. Evans II, P. Caselli, P.C. Myers, Y. Shirley, Y. Aikawa, and M. Tafalla, An Observational Perspective of Low-Mass Dense Cores I: Internal Physical and Chemical Properties, Protostars and Planets V (2007), pp. $17-32$.

[9] M.H. Heyer, J.P. Williams, and C.M. Brunt, Turbulent Gas Flows in the Rosette and G216-2.5 Molecular Clouds: Assessing Turbulent Fragmentation Descriptions of Star Formation, ApJ 643 (2006), pp. 956-964.

[10] T.A. Kuchar and T.M. Bania, A high-resolution H I survey of the Rosette Nebula, ApJ 414 (1993), pp. 664-671.

[11] J.P. Williams, R.K. Mann, C.N. Beaumont, J.J. Swift, J.D. Adams, J. Hora, M. Kassis, E.A. Lada, and C.G. RománZúñiga, Diverse Protostellar Evolutionary States in the Young Cluster AFGL961, ApJL 699 (2009), pp. 1300-1306.

[12] J.P. Williams and R.J. Maddalena, A Large Photodissociation Region around the Cold, Unusual Cloud G216-2.5, ApJ 464 (1996), pp. $247-+$.

[13] L. Hartmann, J. Ballesteros-Paredes, and E.A. Bergin, Rapid Formation of Molecular Clouds and Stars in the Solar Neighborhood, ApJ 562 (2001), pp. 852-868.

[14] C.D. Matzner, On the Role of Massive Stars in the Support and Destruction of Giant Molecular Clouds, ApJ 566 (2002), pp. 302-314.

[15] N.J. Evans et al., The Spitzer c2d Legacy Results: Star-Formation Rates and Efficiencies; Evolution and Lifetimes, ApJS 181 (2009), pp. 321-350.

[16] S.W. Stahler and F. Palla The Formation of Stars, , 2005. 
[17] C.J. Lada and E.A. Lada, Embedded Clusters in Molecular Clouds, ARA\&A 41 (2003), pp. 57-115.

[18] N.Z. Scoville, M. Polletta, S. Ewald, S.R. Stolovy, R. Thompson, and M. Rieke, High-Mass, OB Star Formation in

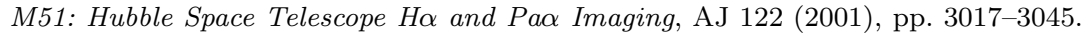

[19] P. Kroupa, On the variation of the initial mass function, MNRAS 322 (2001), pp. 231-246.

[20] K.E. Haisch Jr., E.A. Lada, and C.J. Lada, Disk Frequencies and Lifetimes in Young Clusters, ApJL 553 (2001), pp. L153-L156.

[21] J. Hernández, L. Hartmann, N. Calvet, R.D. Jeffries, R. Gutermuth, J. Muzerolle, and J. Stauffer, A Spitzer View of Protoplanetary Disks in the $\gamma$ Velorum Cluster, ApJ 686 (2008), pp. 1195-1208.

[22] S.M. Andrews and J.P. Williams, Circumstellar Dust Disks in Taurus-Auriga: The Submillimeter Perspective, ApJ 631 (2005), pp. 1134-1160.

[23] ㄴ. A Submillimeter View of Circumstellar Dust Disks in $\rho$ Ophiuchi, ApJ 671 (2007), pp. 1800-1812.

[24] J.M. Carpenter, Constraints on the Circumstellar Disk Masses in the IC 348 Cluster, AJ 124 (2002), pp. 1593-1600.

[25] J. Bally, R.S. Sutherland, D. Devine, and D. Johnstone, Externally Illuminated Young Stellar Environments in the Orion Nebula: Hubble Space Telescope Planetary Camera and Ultraviolet Observations, AJ 116 (1998), pp. 293-321.

[26] M.J. McCaughrean and C.R. O'dell, Direct Imaging of Circumstellar Disks in the Orion Nebula, AJ 111 (1996), pp. $1977-+$.

[27] R.K. Mann and J.P. Williams, The Circumstellar Disk Mass Distribution in the Orion Trapezium Cluster, ApJL 694 (2009), pp. L36-L40.

[28] M. Wadhwa, Y. Amelin, A.M. Davis, G.W. Lugmair, B. Meyer, M. Gounelle, and S.J. Desch, From Dust to Planetesimals: Implications for the Solar Protoplanetary Disk from Short-lived Radionuclides, Protostars and Planets V (2007), pp. $835-848$

[29] A.N. Krot, E.R.D. Scott, and B. Reipurth (eds.), Chapter title. Chondrites and the Protoplanetary Disk, Vol. 341 of Astronomical Society of the Pacific Conference Series (2005),

[30] P.M. Jeffery and J.H. Reynolds, Origin of Excess Xe ${ }^{129}$ in Stone Meteorites, JGR 66 (1961), pp. 3582-3583.

[31] E. Zinner, PLANETARY SCIENCE: An Isotopic View of the Early Solar System, Science 300 (2003), pp. 265-267.

[32] D.N. Schramm and G.J. Wasserburg, Nucleochronologies and the Mean Age of the Elements, ApJ 162 (1970), pp. $57-+$.

[33] A. N. Krot, E. R. D. Scott, \& B. Reipurth (ed.), Chapter title. The Birth of the Solar System in a Molecular Cloud: Evidence from the Isotopic Pattern of Short-lived Nuclides in the Early Solar System, Vol. 341 of Astronomical Society of the Pacific Conference Series (2005),

[34] G.R. Huss, B.S. Meyer, G. Srinivasan, J.N. Goswami, and S. Sahijpal, Stellar sources of the short-lived radionuclides in the early solar system, GCA 73 (2009), pp. 4922-4945.

[35] T. Lee, D.A. Papanastassiou, and G.J. Wasserburg, Aluminum-26 in the early solar system - Fossil or fuel, ApJL 211 (1977), pp. L107-L110.

[36] R. Diehl et al., Radioactive ${ }^{26}$ Al from massive stars in the Galaxy, Nature 439 (2006), pp. 45-47.

[37] S. Sahijpal, P. Soni, and G. Gupta, Numerical simulations of the differentiation of accreting planetesimals with $26 \mathrm{Al}$ and 60Fe as the heat sources, Meteoritics and Planetary Science 42 (2007), pp. 1529-1548.

[38] S.J. Desch and L.A. Leshin, Making Water Worlds: The Role of Al 26, in Lunar and Planetary Institute Science Conference AbstractsS. Mackwell \& E. Stansbery ed., , Vol. 35 of Lunar and Planetary Inst. Technical Report, Mar., 2004, pp. 1987-+

[39] J.D. Gilmour and C.A. Middleton, Anthropic selection of a Solar System with a high ${ }^{26}$ Al/ ${ }^{27}$ Al ratio: Implications and a possible mechanism, Icarus 201 (2009), pp. 821-823.

[40] D. Charbonneau et al., A super-Earth transiting a nearby low-mass star, Nature 462 (2009), pp. 891-894.

[41] A.G.W. Cameron and J.W. Truran, The supernova trigger for formation of the solar system, Icarus 30 (1977), pp. $447-461$.

[42] T. Lee, F.H. Shu, H. Shang, A.E. Glassgold, and K.E. Rehm, Protostellar Cosmic Rays and Extinct Radioactivities in Meteorites, ApJ 506 (1998), pp. 898-912.

[43] K.D. McKeegan, M. Chaussidon, and F. Robert, Incorporation of Short-Lived ${ }^{10}$ Be in a Calcium-Aluminum-Rich Inclusion from the Allende Meteorite, Science 289 (2000), pp. 1334-1337.

[44] G. Rugel, T. Faestermann, K. Knie, G. Korschinek, M. Poutivtsev, D. Schumann, N. Kivel, I. Günther-Leopold, R. Weinreich, and M. Wohlmuther, New Measurement of the Fe60 Half-Life, Physical Review Letters 103 (2009), pp. $072502-+$.

[45] S. Tachibana and G.R. Huss, The Initial Abundance of ${ }^{60} \mathrm{Fe}$ in the Solar System, ApJL 588 (2003), pp. L41-L44.

[46] M. Regelous, T. Elliott, and C.D. Coath, Nickel isotope heterogeneity in the early Solar System, Earth and Planetary Science Letters $272(2008)$, pp. $330-338$

[47] S. Mostefaoui, G.W. Lugmair, and P. Hoppe, ${ }^{60} \mathrm{Fe}$ : A Heat Source for Planetary Differentiation from a Nearby Supernova Explosion, ApJ 625 (2005), pp. 271-277.

[48] S. Tachibana, G.R. Huss, N.T. Kita, G. Shimoda, and Y. Morishita, ${ }^{60}$ Fe in Chondrites: Debris from a Nearby Supernova in the Early Solar System?, ApJL 639 (2006), pp. L87-L90.

[49] M.J. Harris, J. Knödlseder, P. Jean, E. Cisana, R. Diehl, G.G. Lichti, J. Roques, S. Schanne, and G. Weidenspointner, Detection of $\gamma$-ray lines from interstellar ${ }^{60} \mathrm{Fe}$ by the high resolution spectrometer SPI, A\&A 433 (2005), pp. L49-L52.

[50] A.G.W. Cameron, Nucleosynthesis and star formation, in Protostars and Planets IIIE. H. Levy \& J. I. Lunine ed., , 1993, pp. 47-73.

[51] G.J. Wasserburg, M. Busso, R. Gallino, and K.M. Nollett, Short-lived nuclei in the early Solar System: Possible AGB sources, Nuclear Physics A 777 (2006), pp. 5-69.

[52] G. Schaller, D. Schaerer, G. Meynet, and A. Maeder, New grids of stellar models from 0.8 to 120 solar masses at $Z=$ 0.020 and $Z=0.001$, A\&AS 96 (1992), pp. 269-331.

[53] J.H. Kastner and P.C. Myers, An observational estimate of the probability of encounters between mass-losing evolved stars and molecular clouds, ApJ 421 (1994), pp. 605-615.

[54] M. Jura and S.G. Kleinmann, Very dusty carbon-rich asymptotic giant branch stars between about 1 and about 2.5 kiloparsecs from the sun, ApJ 364 (1990), pp. 663-671.

[55] J.P. Williams and C.F. McKee, The Galactic Distribution of OB Associations in Molecular Clouds, ApJ 476 (1997), pp. $166-+$

[56] G. Tenorio-Tagle and P. Bodenheimer, Large-scale expanding superstructures in galaxies, ARA\&A 26 (1988), pp. $145-197$. 
[57] L.W. Looney, J.J. Tobin, and B.D. Fields, Radioactive Probes of the Supernova-contaminated Solar Nebula: Evidence that the Sun Was Born in a Cluster, ApJ 652 (2006), pp. 1755-1762.

[58] M. Limongi and A. Chieffi, The Nucleosynthesis of ${ }^{26} \mathrm{Al}$ and ${ }^{60} \mathrm{Fe}$ in Solar Metallicity Stars Extending in Mass from 11 to $120 M_{\text {solar: }}$ The Hydrostatic and Explosive Contributions, ApJ 647 (2006), pp. 483-500.

[59] D. Jewitt, J. Luu, and C. Trujillo, Large Kuiper Belt Objects: The Mauna Kea 8K CCD Survey, AJ 115 (1998), pp. $2125-2135$.

[60] N. Ouellette, S.J. Desch, and J.J. Hester, Interaction of Supernova Ejecta with Nearby Protoplanetary Disks, ApJ 662 (2007), pp. 1268-1281.

[61] R.A. Chevalier, Young Circumstellar Disks near Evolved Massive Stars and Supernovae, ApJL 538 (2000), pp. L151L154.

[62] H. Zinnecker and H.W. Yorke, Toward Understanding Massive Star Formation, ARA\&A 45 (2007), pp. $481-563$.

63] J.P. Williams and E. Gaidos, On the Likelihood of Supernova Enrichment of Protoplanetary Disks, ApJL 663 (2007), pp. L33-L36.

64] M. Gounelle and A. Meibom, The Origin of Short-lived Radionuclides and the Astrophysical Environment of Solar System Formation, ApJ 680 (2008), pp. 781-792.

65] C.J. Lada and N.D. Kylafis (eds.), Chapter title. The physics of star formation and early stellar evolution (1991),

[66] B.G. Elmegreen and C.J. Lada, Sequential formation of subgroups in OB associations, ApJ 214 (1977), pp. 725-741.

67] J.J. Hester and S.J. Desch, Understanding Our Origins: Star Formation in HII Region Environments, in Chondrites and the Protoplanetary DiskA. N. Krot, E. R. D. Scott, \& B. Reipurth ed.,, Vol. 341 of Astronomical Society of the Pacific Conference Series, Dec., , 2005, pp. 107-+.

[68] B.G. Elmegreen and J. Palous (eds.), Chapter title. Triggered Star Formation in a Turbulent ISM, Vol. 237 of IAU Symposium (2007), .

[69] J.J. Hester et al., Hubble Space Telescope WFPC2 Imaging of M16: Photoevaporation and Emerging Young Stellar Objects, AJ 111 (1996), pp. 2349-+.

[70] H.A.T. Vanhala and A.P. Boss, Injection of Radioactivities into the Forming Solar System, ApJ 575 (2002), pp. $1144-1150$.

[71] G. Mellema, S.J. Arthur, W.J. Henney, I.T. Iliev, and P.R. Shapiro, Dynamical H II Region Evolution in Turbulent Molecular Clouds, ApJ 647 (2006), pp. 397-403.

[72] T. Freyer, G. Hensler, and H.W. Yorke, Massive Stars and the Energy Balance of the Interstellar Medium. I. The

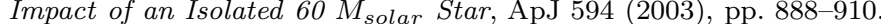

[73] W.J. Lang, M.R.W. Masheder, T.M. Dame, and P. Thaddeus, The lambda-Orionis Ring in CO, A\&A 357 (2000), pp. $1001-1012$.

[74] C.N. Beaumont and J.P. Williams, Molecular Rings Around Interstellar Bubbles and the Thickness of Star-Forming Clouds, ApJ 709 (2010), pp. 791-800.

[75] A.P. Boss, S.A. Keiser, S.I. Ipatov, E.A. Myhill, and H.A.T. Vanhala, Triggering Collapse of the Presolar Dense Cloud Core and Injecting Short-lived Radioisotopes with a Shock Wave. I. Varied Shock Speeds, ApJ 708 (2010), pp. $1268-1280$.

[76] L. Deharveng, A. Zavagno, F. Schuller, J. Caplan, M. Pomarès, and C. De Breuck, Star formation around RCW 120, the perfect bubble, A\&A 496 (2009), pp. 177-190.

[77] E. Gaidos, A.N. Krot, J.P. Williams, and S.N. Raymond, ${ }^{26}$ Al and the Formation of the Solar System from a Molecular Cloud Contaminated by Wolf-Rayet Winds, ApJ 696 (2009), pp. 1854-1863.

[78] U. Hwang et al., A Million Second Chandra View of Cassiopeia A, ApJL 615 (2004), pp. L117-L120.

[79] M. Gounelle, A. Meibom, P. Hennebelle, and S. Inutsuka, Supernova Propagation and Cloud Enrichment: A New Model for the Origin of ${ }^{60} \mathrm{Fe}$ in the Early Solar System, ApJL 694 (2009), pp. L1-L5.

[80] J. Maza and S. van den Bergh, Statistics of extragalactic supernovae, ApJ 204 (1976), pp. 519-529.

[81] R.J. Rand and S.R. Kulkarni, M51 - Molecular spiral arms, giant molecular associations, and superclouds, ApJL 349 (1990), pp. L43-L46.

[82] R. Shetty and E.C. Ostriker, Cloud and Star Formation in Disk Galaxy Models with Feedback, ApJ 684 (2008), pp. 978-995.

[83] J. Koda et al., Dynamically Driven Evolution of the Interstellar Medium in M51, ApJL 700 (2009), pp. L132-L136.

84] R.A. Scheepmaker, H.J.G.L.M. Lamers, P. Anders, and S.S. Larsen, The spatial distribution of star and cluster formation in M 51, A\&A 494 (2009), pp. 81-93.

[85] E.D. Young, K.M. Pontoppidan, R.L. Smith, M.R. Morris, and M. Gounelle, The Oxygen Isotopic Case for Supernova Enrichment of the Solar System Birth Environment, in Lunar and Planetary Institute Science Conference Abstracts, Vol. 41 of Lunar and Planetary Inst. Technical Report, Mar., , 2010, pp. 1550-+.

[86] C.L. Harper Jr., Astrophysical Site of the Origin of the Solar System Inferred from Extinct Radionuclide Abundances, ApJ 466 (1996), pp. 1026-+. 